\title{
Componentes de (Co)Variância e Parâmetros Genéticos para Caracteres Produtivos à Desmama de Bezerros Angus Criados no Estado do Rio Grande do Sul ${ }^{1}$
}

\author{
Fernando Flores Cardoso², Ricardo Alberto Cardellino ${ }^{3}$, Leonardo Talavera Campos 4
}

\begin{abstract}
RESUMO - Foram estimados componentes de (co)variância e parâmetros genéticos para peso ao nascer (PN), ganho do nascimento à desmama (G205) e escores de conformação (CD), precocidade de terminação (GD), musculatura (MD) e tamanho (TD) à desmama, utilizando-se registros de desmama de 40.915 bezerros Angus, criados no Estado do Rio Grande do Sul, sul do Brasil. Desses, 12.706 tinham pesagem ao nascer e 11.863, avaliação completa para escores visuais (EV). Os dados foram analisados por meio de um modelo animal, em análises uni e multivariadas, e os componentes de variância estimados pela máxima verossimilhança restrita. As herdabilidades aditivas diretas estimadas foram 0,$29 ; 0,25 ; 0,18 ; 0,19 ; 0,19 ; \mathrm{e} 0,21$, respectivamente, para PN, G205, CD, GD, MD e TD. A herdabilidade materna para G205 foi 0,16 e a correlação entre efeito genético direto e materno, -0,51. Essa correlação negativa indica antagonismo entre esses efeitos e provocou decréscimo na herdabilidade total para G205, que foi 0,18 . A contribuição do ambiente permanente da vaca para a variância fenotípica esteve entre um mínimo de 0,05 para PN e máximo de 0,12 para G205. A correlação genética entre PN e G205 foi $-0,06$, mostrando que estes caracteres são independentes geneticamente. As correlações genéticas encontradas entre G205 e EV foram entre 0,71 e 0,86 e de EV entre si, 0,58 a 0,91. Essas associações positivas entre os escores visuais e destes com o crescimento na fase pré-desmama favorecem a seleção conjunta destes caracteres, por meio de índices de seleção.
\end{abstract}

Palavras-chave: bovinos de corte, correlações, herdabilidade, modelo animal

\section{(Co)Variance Components and Genetic Parameters for Weaning Production Traits of Angus Calves Raised in the State of Rio Grande do Sul}

\begin{abstract}
Co) variance components and genetic parameters for birth weight (BW), adjusted weaning gain (AWG) and for conformation (WC), precocity (WP), muscling (WM) and size (WS) scores at weaning were estimated, from records of 40,915 Angus calves, raised in the state of Rio Grande do Sul, southern Brazil. From that data, 12,706 had birth weight records and 11,863 had complete records for visual scores (VS). The data were analyzed using an animal model, in uni and multivariate analyses, and the variance components estimated by restrict maximum likelihood. The direct additive heritabilities estimated were $0.29,0.25,0.18,0.19,0.19$ and 0.21 , respectively for BW, AWG, WC, WP, WM and WS. Maternal heritability for AWG was 0.16 and genetic correlation between direct and maternal effects was -0.51 . This high negative correlation indicates antagonism between these effects and decreases total heritability for AWG to 0.18 . The contribution of dam permanent environment to phenotypic variance was between a minimum of 0.05 for BW and a maximum of 0.12 for AWG. Genetic correlation between BW and AWG was -0.06, showing that these traits are genetically nondependent. The genetic correlations found between AWG and VS were from 0.71 to 0.86 , and among VS traits from 0.58 to 0.91 . These positive associations among VS traits and these with pre-weaning growth phase, support the simultaneous selection of these traits, by means of selection indexes.
\end{abstract}

Key Words: animal model, beef cattle, correlation, heritability

\section{Introdução}

O desempenho pré-desmama de bovinos é influenciado pelo genótipo do indivíduo (efeito genético direto), pelo genótipo da mãe para caracteres maternais e pelos correspondentes efeitos ambientais materno permanente e temporário (HOHENKOKEN e BRINKS, 1971; WILLHAM, 1972). Muitos estudos têm mostrado existência de antagonismo entre os componentes genéticos direto e materno (MEYER, 1992; ROBINSON, 1996a). Para otimizar o progresso genético até a fase de desmama, nos programas de melhoramento, ambos componentes, direto e materno, devem ser considerados, pois a correlação genética negativa entre estes dois componentes pode fazer com que grande parte do ganho obtido em determinada

\footnotetext{
${ }^{1}$ Parte da Dissertação apresentada pelo primeiro autor à FAEM/UFPEL, para obtenção do título de Mestre em Ciências. Financiada pelas CAPES.

2 Aluno de doutorado. Department of Animal Science. Michigan State University. Bolsista da CAPES. E-mail: cardosof@msu.edu

3 Professor do Departamento de Zootecnia FAEM/UFPEL. CEP 96010-900 Pelotas/RS. Bolsista do CNPq. E.mail: rcard@ufpel.tche.br

${ }^{4}$ Associação Nacional de Criadores "Herd Book Collares". E-mail: Itcampos@terra.com.br
} 
geração seja anulada na geração seguinte, pela redução na habilidade materna (ELER et al., 1989).

O peso ao nascer e o ganho de peso têm sido os principais caracteres avaliados até a fase de desmama. Entretanto, no momento atual, visando selecionar animais que atendam às exigências de mercado, com boas características de carcaça, acabamento precoce para o abate sem tamanho maduro excessivo, escores visuais de avaliação para conformação, precocidade de terminação, musculatura e tamanho têm sido também considerados (CAMPOS e CARDOSO, 1995; FRIES, 1996).

A escolha do método de seleção e o progresso genético dependem do conhecimento dos parâmetros genéticos (herdabilidades e correlações genéticas) das populações. Há uma marcada diferença nestes parâmetros entre populações criadas em ambientes diferentes (KOOTS et al., 1994a,b). Estimativas confiáveis de parâmetros genéticos das populações locais são necessárias à adequada condução dos programas de melhoramento genético em cada região. Recentemente, alguns autores têm utilizado modelos animais para estimativas dos efeitos genéticos direto e materno, em bovinos Nelore no Brasil (ELER, et al., 1995; MERCADANTE e LOBO, 1997). A literatura nacional, contudo, ainda carece de estudos de parâmetros genéticos em bovinos de origem taurina, considerando efeitos genéticos direto e materno. Os trabalhos encontrados que estimam herdabilidades e correlações genéticas para escores visuais são poucos e sem resultados conclusivos (MOHIUDDIN, 1993; ROSO e FRIES, 1995).

Os objetivos deste estudo foram estimar componentes de variância e parâmetros genéticos para peso ao nascer, ganho do nascimento à desmama e para escores de conformação, precocidade de terminação, musculatura e tamanho à desmama e determinar as correlações genéticas, ambientais e fenotípicas entre peso ao nascere ganho do nascimento à desmama, do ganho do nascimento à desmama com escores de avaliação visual à desmama e dos escores de avaliação visual entre si.

\section{Material e métodos}

O trabalho foi realizado com dados de campo obtidos da Associação Nacional de Criadores "Herd Book Collares" - ANC, pertencentes a rebanhos da raça Angus controlados pelo Programa de Melhoramento de Bovinos de Carne - PROMEBO, durante os anos de 1974 a 1997. Nas análises, foram considera- dos o peso ao nascer (PN), o ganho de peso ajustado para 205 dias (G205) e escores de avaliação visual (EV). O G205 foi calculado a partir dos pesos ao nascer e à desmama, seguindo-se as normas da BEEF IMPROVEMENT FEDERATION (1996). Para animais sem registro de peso ao nascer foram utilizados pesos padrão ao nascer ( $32 \mathrm{~kg}$ para machos e $30 \mathrm{~kg}$ para fêmeas). Os caracteres avaliados pelos escores consistem em: conformação (CD), que estima a quantidade de carne na carcaça do animal, quando este for abatido, pelo comprimento e profundidade do corpo do animal, aliado ao desenvolvimento muscular e harmonia geral do indivíduo; precocidade de terminação (GD), que estima a capacidade de armazenar gordura, indicando rapidez para atingir o acabamento; musculatura (MD), que representa o grau de desenvolvimento muscular apresentado pelo animal; e tamanho (TD), que compreende o comprimento e a altura do indivíduo. As avaliações foram feitas em uma escala de 1 a 5 , em que cinco representa o grau máximo de expressão da característica, relativa ao grupo contemporâneo.

Foram utilizados dados de 40.915 animais com registros de desmama, dos quais 12.706 também tinham pesagem ao nascer. Foram considerados para a análise de escores visuais somente registros com avaliações completas para os quatro escores e coletados a partir de 1989, totalizando 11.863 produtos. Grupos contemporâneos com menos de dez animais e touros com menos de cinco filhos foram previamente excluídos da análise, resultando na eliminação da base de dados de 918 registros de PN, 1.950 de G205 e 526 escores visuais. Foram formados três arquivos separados para PN, G205 e EV. A descrição dos dados nestes três arquivos é apresentada na Tabela 1.

Todas as informações disponíveis de genealogia foram utilizadas principalmente de touros pais, obtidas porextensa pesquisa nos registros do"Herd BookCollares".

Os efeitos fixos considerados nos modelos foram definidos conforme resultados encontrados por CARDOSO (1999), em outros estudos realizados com o mesmo conjunto de dados. O modelo mais completo utilizado foi o seguinte:

$$
\mathrm{y}=\mu+\mathrm{X} \beta+\mathrm{Zg}+\mathrm{Mm}+\mathrm{Wp}+\mathrm{e},
$$

em que y é o vetor de observações; $\mu$, a média geral; $\beta$, o vetor de efeitos fixos (grupo contemporâneo ano, estação, rebanho, manejo e data da pesagem; mês de nascimento, idade da vaca, sexo e idade do bezerro); $X$, a matriz de incidência dos efeitos fixos; $\mathrm{g}$, o vetor de efeitos genéticos aditivos diretos; $\mathrm{Z}$, a matriz de incidência dos efeitos genéticos aditivos diretos; 
Rev. bras. zootec.

Tabela 1 - Descrição dos dados de desempenho utilizados na estimação dos componentes de variância Table 1 - Description of performance data used in the estimation of variance components

\begin{tabular}{|c|c|c|c|c|c|c|}
\hline $\begin{array}{l}\text { Caráter } \\
\text { Trait } \\
\end{array}$ & $\begin{array}{c}\text { Registros } \\
\text { Records }\end{array}$ & $\begin{array}{l}\text { Animais }{ }^{1} \\
\text { Animals }^{1}\end{array}$ & $\begin{array}{l}\mathrm{GC}^{2} \\
C G^{2} \\
\end{array}$ & $\begin{array}{c}\text { Touros } \\
\text { Sires }\end{array}$ & $\begin{array}{l}\text { Vacas } \\
\text { Dams }\end{array}$ & $\begin{array}{c}\text { Média } \pm \mathrm{DP} \\
\text { Mean } \pm S D\end{array}$ \\
\hline $\begin{array}{l}\text { Peso ao nascer (kg) } \\
\text { Birth weight }\end{array}$ & 11.788 & 22.656 & 221 & 326 & 8506 & $32,87 \pm 4,36$ \\
\hline $\begin{array}{l}\text { Ganho desmama }(\mathrm{kg}) \\
\text { Weaning gain }\end{array}$ & 38.965 & 61.216 & 676 & 791 & 22.283 & $124,09 \pm 32,78$ \\
\hline $\begin{array}{l}\text { Conformação (un.) } \\
\text { Conformation }\end{array}$ & 11.337 & 20.200 & 170 & 297 & 7701 & $2,92 \pm 1,10$ \\
\hline $\begin{array}{l}\text { Precocidade (un.) } \\
\text { Precocity }\end{array}$ & 11.337 & 20.200 & 170 & 297 & 7701 & $3,09 \pm 1,08$ \\
\hline $\begin{array}{l}\text { Musculatura (un.) } \\
\text { Muscling }\end{array}$ & 11.337 & 20.200 & 170 & 297 & 7701 & $2,97 \pm 1,06$ \\
\hline $\begin{array}{l}\text { Tamanho (un.) } \\
\text { Size }\end{array}$ & 11.337 & 20.200 & 170 & 297 & 7701 & $3,00 \pm 1,06$ \\
\hline
\end{tabular}

${ }^{1}$ Número total de animais em $\mathrm{A}^{-1}$, incluindo animais sem registros. ${ }^{2}$ Grupos contemporâneos.

1 Total number of animals in $A^{-1}$, including animals without records. ${ }^{2}$ Contemporary groups.

$\mathrm{m}$, o vetor de efeitos genéticos aditivos maternos; $\mathrm{M}$, a matriz de incidência dos efeitos genéticos aditivos maternos; $p$, o vetor de efeitos de ambiente materno permanente; $\mathrm{W}$, a matriz de incidência dos efeitos de ambiente materno permanente; $\mathrm{e}, \mathrm{o}$ efeito residual. Para este modelo são válidas as seguintes condições:

$$
\begin{aligned}
& \mathrm{E}[\mathrm{y}]=\mathrm{X} \beta \\
& E\left[\begin{array}{c}
g \\
m \\
p \\
e
\end{array}\right]=\left[\begin{array}{l}
0 \\
0 \\
0 \\
0
\end{array}\right] \operatorname{Var}\left[\begin{array}{l}
g \\
m \\
p \\
e
\end{array}\right]=\left[\begin{array}{cccc}
A \sigma_{g}^{2} & A \sigma_{g m} & 0 & 0 \\
A \sigma_{g m} & A \sigma_{m}^{2} & 0 & 0 \\
0 & 0 & I_{n c} \sigma_{p}^{2} & 0 \\
0 & 0 & 0 & I_{n} \sigma_{e}^{2}
\end{array}\right]
\end{aligned}
$$

em que nc é o número de vacas e n, o número de observações; A, a matriz de parentesco e I, as matrizes identidade; $\sigma_{\mathrm{g}}^{2}$, a variância genética aditiva direta; $\sigma_{m}^{2}$, a variância genética aditiva materna; $\sigma_{\mathrm{gm}}$, a covariância genética entre efeitos aditivos direto e materno; $\sigma_{c}^{2}$, a variância de ambiente permanente; e $\sigma_{\text {e }}^{2}$, a variância ambiental.

O termo Mm do modelo geral (efeito genético materno) não foi incluído na estimação dos parâmetros para PN e EV, pois mostrou-se sem importância significativa em análise prévia dos dados, com herdabilidades próximas a zero. O termo $\mathrm{Wp}$ do modelo geral foi mantido com o fim de considerar os efeitos maternos permanentes sobre estes caracteres.

Os componentes de variância e covariância foram estimados por meio da máxima verossimilhança restrita livre de derivadas (DFREML), em análises uni e multivariadas, utilizando-se o programa MTDFREML (BOLDMAN et al., 1995).

Inicialmente, análises univariadas foram realizadas para todos os caracteres estudados. Os componentes de variância obtidos nestas análise foram utilizados como valores iniciais para as análises multivariadas. Os caracteres foram analisados em pares: PN com G205; G205 com todos os EV; e EV entre si. Valores perdidos não foram considerados nas análises, em parte por razões computacionais, mas também porque dentro de um grupo contemporâneo, se há pesagem ao nascer, normalmente todos os animais são pesados. O mesmo ocorre com EV em cujo caso há duas opções: ou todos os animais do grupo são avaliados ou nenhum animal é avaliado. Desse modo, os caracteres estudados estão livres de efeitos de pré-seleção.

Médias dos valores foram calculados para os componentes de variância e parâmetros genéticos, obtidos a partir das análises bivariadas. A herdabilidade para mérito genético total (direto + materno) em G205 foi calculada como proposto por WILLHAM (1972), por meio da seguinte fórmula:

$$
\mathrm{h}^{2}{ }_{\mathrm{t}}=\left(\sigma_{\mathrm{g}}^{2}+0,5 \sigma_{\mathrm{m}}^{2}+1,5 \sigma_{\mathrm{gm}}\right) / \sigma_{\mathrm{p}}^{2}
$$

em que $\sigma_{p}^{2}$ é a variância fenotípica, dada pela seguinte expressão:

$$
\sigma_{\mathrm{p}}^{2}=\sigma_{\mathrm{g}}^{2}+\sigma_{\mathrm{m}}^{2}+\sigma_{\mathrm{gm}}+\sigma_{\mathrm{c}}^{2}+\sigma_{\mathrm{e}}^{2}
$$

\section{Resultados e Discussão}

As estimativas dos componentes de variância e os parâmetros genéticos para os caracteres estudados, a partir de modelos univariados, são apresentados na Tabela 2 .

A magnitude das herdabilidades foi média para todos os caracteres estudados. O maior valor foi observado em PN, 0,29. Não foi incluído no modelo o efeito genético aditivo materno na análise deste caráter, entretanto, o efeito materno permanente foi con- 
siderado. A variância genética aditiva para PN neste conjunto de dados foi superior à encontrada por SCARPATI et al. (1998), analisando dados da raça Nelore, com o mesmo modelo, mas está abaixo da encontrada por MEYER (1992). Ambos pesquisadores constataram que a inclusão do efeito genético materno não afetou a herdabilidade total, quando o efeito de ambiente permanente da vaca já estava contemplado no modelo. WILSON et al. (1986) encontraram herdabilidade do PN mais baixa $(0,19) \mathrm{em}$ animais Angus, nos Estados Unidos, e suspeitaram que muitos registros de PN foram inacuradamente estimados pelos criadores. Apesar das dificuldades associadas à medida de $\mathrm{PN}$ em condições extensivas de criação, a herdabilidade encontrada neste estudo é praticamente igual à média da literatura (KOOTS et al., 1994a) e indica que progresso genético pode ser alcançado, pela seleção por desempenho individual, na população da raça Angus do RS.
Para G205 a estimativa de 0,25 para herdabilidade aditiva direta está abaixo da média das estimativas encontrada na literatura internacional (KOOTS et al., 1994a; MEYER, 1992), mas está de acordo com estudos realizados a partir de dados de campo do RS (FRIES, 1984; PONS et al., 1989) e de outros estados do Brasil (MERCADANTE e LOBO, 1997) e com os encontrados por ROBINSON (1996a), na Austrália, e por WILSON et al. (1986), nos Estados Unidos. Esses dois últimos estudos foram baseados em dados de campo de animais Angus e resultaram em valores de herdabilidade entre 0,15 e 0,26 para o crescimento pré-desmama.

A herdabilidade aditiva materna para G205 foi 0,16 , indicando que é possível aumentar o crescimento pré-desmama pela seleção de fêmeas de maior habilidade materna. Entretanto, pode-se observar a existência de antagonismo entre os efeitos genéticos direto e materno no G205, pois a correlação genética

Tabela 2 - Estimativas dos componentes de variância e parâmetros genéticos para peso ao nascer (PN), ganho de peso do nascimento à desmama ajustado a 205 dias (G205), conformação (CD), precocidade (GD), musculatura (MD) e tamanho (TD) à desmama, a partir de análises univariadas

Table 2 - Estimates of variance components and genetic parameters for birth weight (BW), 205 day adjusted weaning gain (AWG), conformation (WC), precocity (WP), muscling (WM) and size (WS) at weaning, from univariate analyses

\begin{tabular}{lllllll}
\hline Caraterística & PN & G205 & CD & GD & MD & TD \\
Trait & $B W$ & $A W G$ & $W C$ & $W P$ & $W M$ & $W S$ \\
\hline
\end{tabular}

Componentes de variância ${ }^{1,2}$

Variance components ${ }^{1,2}$

$\sigma^{2}$

$\sigma_{m}^{2}$

$\sigma_{\text {gm }}$

$\sigma_{c}^{2}$

$\sigma_{\mathrm{e}}^{2}$

$\sigma^{2}{ }_{\mathrm{t}}$

Parâmetros genéticos ${ }^{3}$

Genetic parameters ${ }^{3}$

$\mathrm{h}^{2} \mathrm{~g}$

$\mathrm{h}^{2} \mathrm{~m}$

$\mathrm{r}_{\mathrm{gm}}$

$c^{2}$

$\mathrm{e}^{2}$

$\mathrm{h}^{2} \mathrm{t}$
3,97

111,42

70,39

$-45,22$

0,71

8,80

13,48

51,10

251,10

438,78

0,29

0,25

0,16

$-0,51$

0,05

0,65

0,29

0,12

0,57

0,18
0,15

0,18

0,17

0,18

0,08

0,64

0,87

0,18

0,19

0,08

0,67

0,93

0,07

0,65

0,89

0,58
0,84
${ }^{1} \mathrm{Em} \mathrm{kg}^{2}$ para PN e G205 e em unidades ${ }^{2}$ para CD, GD, MD e TD. ${ }^{2} \sigma^{2}{ }_{\mathrm{g}}=$ variância genética aditiva direta, $\sigma^{2}{ }_{\mathrm{m}}=$ variância genética aditiva materna, $\sigma_{\mathrm{gm}}=$ covariância genética entre efeitos aditivos direto e materno, $\sigma^{2}{ }_{\mathrm{c}}=$ variância de ambiente permanente, $\sigma^{2}{ }_{\mathrm{e}}^{\mathrm{m}}=$ variância ambiental e $\sigma^{2}{ }_{\mathrm{t}}=$ variância fenotípica. ${ }^{3} \mathrm{~h}^{2} \mathrm{~g}=$ herdabilidade aditiva direta, $\mathrm{h}_{\mathrm{m}}^{2}=$ herdabilidade aditiva materna, $\mathrm{r}_{\mathrm{gm}}=$ correlação genética entre efeitos aditivos direto e materno, $\mathrm{c}^{2}=$ porção da variância devido ao ambiente permanente, $\mathrm{e}^{2}=$ porção da variância devido ao ambiente temporário, $\mathrm{e} \mathrm{h}^{2}{ }_{t}=$ herdabilidade total.

1 In $\mathrm{kg}^{2}$ for BW and $A W G$ and in units ${ }^{2}$ for WC, WP, WM and WS. ${ }^{2} \sigma^{2}{ }_{g}=$ direct additive genetic variance, $\sigma^{2}{ }_{m}=$ maternal additive genetic variance, $\sigma_{g m}=$ covariance between direct and maternal additive genetic effects, $\sigma^{2}{ }_{c}=$ permanent environmental variance, $\sigma^{2}{ }_{e}=$ environmental variance, and $\sigma^{2}{ }_{t}=$ phenotypic variance. ${ }^{3} h^{2}{ }_{g}=$ direct additive heritability, $h^{2}{ }_{m}=$ maternal additive heritability, $r_{g m}=$ genetic correlation between direct and maternal additive genetic effects, $c^{2}=$ fraction of variance due to permanent environmental effects, $e^{2}=$ fraction of variance due to temporary environmental effects, and $h^{2}{ }_{t}=$ total heritability. 
Rev. bras. zootec.

entre estes dois efeitos foi -0,51. Isso reflete em menor ganho genético total esperado em G205 do que $\mathrm{o}$ indicado pela herdabilidade aditiva direta, uma vez que a herdabilidade total observada foi $28 \%$ menor que a herdabilidade genética direta. Resultados semelhantes foram observados em diversos outros estudos (ELER et al., 1989; MEYER, 1992 e 1993). Entretanto, a origem completamente genética desta correlação é questionada e alguns fatores não genéticos, como covariância negativa entre progênie e mãe e interações de touro com ano, são sugeridos como fatores que contribuem, pelo menos em parte, para a natureza negativa desta correlação (ROBINSON, 1996b). Assim, muitos programas de melhoramento tem fixado esta covariância como sendo zero, nas avaliações genéticas.

As estimativas dos componentes de variância e das herdabilidades para EV (Tabela 2) foram muito consistentes e semelhantes entre si. Os valores encontrados de 0,$18 ; 0,19 ; 0,19$; e 0,21 para CD, GD, MD e TD, respectivamente, foram semelhantes aos encontrados para herdabilidade total de G205 $(0,18)$ na mesma população, indicando existência de variação genética aditiva para seleção e progresso nestes caracteres. Os valores de herdabilidade encontrados para EV foram inferiores aos encontrados por outros autores para escores visuais, usados na avaliação de características de carcaça e terminação (ROSO e FRIES, 1995), que estiveram entre 0,18 e 0,27 , mas foram superiores aos observados por CARDOSO et al. (1998) com dados de bovinos Santa Gertrudis criados no Estado do Rio Grande do Sul (entre 0,10 e 0,20).

Outros estudos com escore de conformação em animais Hereford, criados no RS, encontraram herdabilidades entre 0,32 e 0,37 (CARDELLINO e CARDELLINO, 1984; PONS et al., 1989). Entretanto, esses estudos utilizaram um modelo de touro e a covariância entre meios irmãos paternos dentro de touros, sem considerar efeitos maternos, para as estimativas de herdabilidade, o que tende a inflacionar as estimativas da variância genética aditiva (MEYER, 1992). ROBINSON et al. (1993) encontraram herdabilidade de 0,17 para escore de musculatura em animais Angus, na Austrália, e KOCH et al. (1995) obtiveram resposta genética direta favorável em escore de musculatura, quando se selecionou por um índice com 50\% para ganho final e 50\% para escore de musculatura, sem, entretanto, encontrar resposta materna importante neste caráter.

Os resultados deste estudo indicam que os escores visuais, apesar de terem natureza subjetiva, quando aplicados de maneira criteriosa e por avaliadores qualificados, podem servirpara alteraro valor genético dos animais em caracteres relacionados com a carcaça, como grau de desenvolvimento musculare grau de acabamento.

A fração da variância fenotípica devida ao ambiente permanente da vaca em G205 foi até $12 \%$, concordando com o encontrado por ROBINSON (1996a) em animais Angus na Austrália. MEYER (1993) e MEYER et al. (1993) ponderaram que a importância desse componente aumenta em raças como Hereford, em que o potencial de produção de leite da mãe é limitante ao crescimento dos bezerros. Para PN esta fração foi de $5 \%$, indicando que haveria pequena vantagem em aguardar mais de uma produção para selecionar ventres, com respeito a esse caráter. No caso de EV, os valores encontrados (entre 0,08 e 0,09) foram intermediários entre PN e G205, indicando que o ambiente permanente da vaca tem efeito moderado sobre estes caracteres.

As médias para os componentes de variância estimados a partir da análises bivariadas são apresentadas na Tabela 3.

Os valores representam médias de quatro análises, para todos os casos e são consistentes com os observados nas análises univariadas. Houve pequeno incremento da variação total e genética aditiva para G205, sem, contudo, afetar significativamente a herdabilidade genética direta. O valor encontrado para correlação entre efeito genético direto e materno em G205 não foi tão negativo, em comparação com o valor encontrado na análise univariada, resultando em aumento de cinco pontos percentuais na herdabilidade total. As herdabilidades para EV, nas análises multivariadas, permaneceram praticamente inalteradas, tendo incremento médio de $1 \%$ e discordando dos resultados encontrados por CARDOSO et al. (1998), em que houve incremento importante das herdabilidades, nas análise bivariadas de ganho de peso e escores visuais, para ambos os caracteres. Os resultados deste trabalho indicam que modelos univariados são adequados para a análise de EV na fase de desmama, na qual não há pré-seleção dos animais que participam da avaliação.

$\mathrm{Na}$ Tabela 3 não são apresentados valores de componentes de variância para PN, pois uma única análise bivariada foi realizada para este caráter, conjuntamente com G205. Não houve variação importante nas estimativas dos componentes de variância para PN, entre a análise bivariada com G205 e a análise univariada (Tabela 2); assim, os modelos univariados também são adequados para as análises de PN. 
Tabela 3 - Médias das estimativas dos componentes de variância e parâmetros genéticos para peso ao nascer (PN), ganho de peso do nascimento à desmama ajustado a 205 dias (G205), conformação (CD), precocidade (GD), musculatura (MD) e tamanho (TD) à desmama, a partir de análises multivariadas

Table 3 - Average estimates of variance components and genetic parameters for birth weight (BW), 205 day adjusted weaning gain (AWG), conformation (WC), precocity (WP), muscling (WM) and size (WS) at weaning, from multivariate analyses

\begin{tabular}{|c|c|c|c|c|c|}
\hline $\begin{array}{l}\text { Caraterística } \\
\text { Trait }\end{array}$ & $\begin{array}{l}\mathrm{G} 205 \\
A W G\end{array}$ & $\begin{array}{l}\mathrm{CD} \\
W C\end{array}$ & $\begin{array}{l}\mathrm{GD} \\
W P\end{array}$ & $\mathrm{MD}$ & $\begin{array}{l}\mathrm{TD} \\
W S\end{array}$ \\
\hline \multicolumn{6}{|c|}{ Componentes de variância ${ }^{1,2}$} \\
\hline $\begin{array}{l}\text { Variance comp } \\
\sigma_{\mathrm{g}}^{2}\end{array}$ & 127,86 & 0,17 & 0,19 & 0,17 & 0,18 \\
\hline$\sigma_{\mathrm{m}}^{2}$ & 59,70 & & & & \\
\hline$\sigma_{\mathrm{gm}}$ & $-30,48$ & & & & \\
\hline$\sigma_{c}^{2}$ & 56,04 & 0,07 & 0,08 & 0,07 & 0,08 \\
\hline$\sigma_{\mathrm{e}}^{2}$ & 276,72 & 0,63 & 0,66 & 0,64 & 0,58 \\
\hline$\sigma_{t}^{2}$ & 489,84 & 0,87 & 0,93 & 0,89 & 0,84 \\
\hline \multicolumn{6}{|c|}{ Parâmetros genéticos 3} \\
\hline \multicolumn{6}{|c|}{ Genetic parameters ${ }^{3}$} \\
\hline$h^{2}{ }_{g}$ & 0,26 & 0,20 & 0,21 & 0,19 & 0,22 \\
\hline $\mathrm{h}_{\mathrm{m}}^{2}$ & 0,12 & & & & \\
\hline$r_{\text {gm }}$ & $-0,35$ & & & & \\
\hline$c^{2}$ & 0,11 & 0,09 & 0,09 & 0,08 & 0,10 \\
\hline$e^{2}$ & 0,56 & 0,72 & 0,71 & 0,72 & 0,69 \\
\hline $\mathrm{h}^{2}{ }_{\mathrm{t}}$ & 0,23 & 0,20 & 0,21 & 0,19 & 0,22 \\
\hline
\end{tabular}

${ }^{1} \mathrm{Em} \mathrm{kg}^{2}$ para G205 e em unidades ${ }^{2}$ para CD, GD, MD e TD. ${ }^{2} \sigma_{\mathrm{g}}^{2}=$ variância genética aditiva direta, $\sigma_{\mathrm{m}}^{2}=$ variância genética aditiva materna, $\sigma_{\mathrm{gm}}=$ covariância genética entre efeitos aditivos direto e materno, $\sigma^{2}{ }_{\mathrm{c}}=$ variância de ambiente permanente, $\sigma^{2}{ }_{\mathrm{e}}=$ variância ambiental e $\sigma^{2}=$ variância fenotípica. ${ }^{3} \mathrm{~h}_{\mathrm{g}}=$ herdabilidade aditiva direta, $\mathrm{h}_{\mathrm{m}}{ }_{\mathrm{m}}=$ herdabilidade aditiva materna, $\mathrm{r}_{\mathrm{gm}}=$ correlação genética entre efeitos aditivos direto e materno, $\mathrm{c}^{2}=$ porção da variância devida ao ambiente permanente, $\mathrm{e}^{2}=$ porção da variância devida ao ambiente temporário, $\mathrm{e} \mathrm{h}_{\mathrm{t}}{ }_{\mathrm{t}}=$ herdabilidade total. ${ }^{1}$ In kg ${ }^{2}$ for $A W G$ and in units ${ }^{2}$ for WC, WP, WM and WS. ${ }^{2} \sigma^{2}{ }_{g}=$ direct additive genetic variance, $\sigma^{2}{ }_{m}=$ maternal additive genetic variance, $\sigma_{\text {gm }}=$ covariance between direct and maternal additive genetic effects, $\sigma_{c}^{2}{ }_{c}=$ permanent environmental variance, $\sigma^{2}{ }_{e}=$ environmental variance, and $\sigma^{2}{ }_{t}=$ phenotypic variance. ${ }^{3} h^{2}{ }_{g}=$ direct additive heritability, $h_{m}^{2}=$ maternal additive heritability, $r_{g m}=$ genetic correlation between direct and maternal additive genetic effects, $c^{2}=$ fraction of variance due to permanent environmental effects, $e^{2}=$ fraction of variance due to temporary environmental effects, and $h^{2}{ }_{t}=$ total heritability.

Os componentes de covariância e as correlações genéticas entre PN e G205, entre G205 e EV e entre EV são apresentadas na Tabela 4.

A correlação genética de -0,06 entre PN e G205 mostra que esses caracteres são independentes geneticamente e a seleção para maior ganho de peso do nascimento à desmama não implica necessariamente em incremento de PN. Outros autores também encontraram correlações baixas entre PN e peso à desmama (ELER et al., 1995), entretanto, MEYER (1993) e ROBINSON (1996a), trabalhando, respectivamente, com dados de Hereford e Angus, na Austrália, encontraram correlações genéticas de alta magnitude (0,56 e 0,61, respectivamente). Entretanto, como o PN é parte integrante do peso à desmama, e não do ganho pré-desmama, espera-se que as correlações entre $\mathrm{PN}$ e peso à desmama sejam maiores do que entre PN e ganho pré-desmama. A média encontrada na literatura por KOOTS et al. (1994b), a partir de 31 estudos, para correlação genética entre PN e ganho pré-desmama foi de $36 \%$, ainda indicando associação positiva entre esses caracteres, porém de menor intensidade. Esses resultados suportam o uso do ganho pré-desmama em substituição ao peso à desmama para seleção de crescimento nesta fase, pois assim o aumento direto do PN dos animais poderia ser evitado, o que seria vantajoso do ponto de vista da facilidade de parto.

As correlações de ambiente permanente da vaca entre todos os caracteres estudados foram praticamente um, indicando, como em outros estudos (MEYER, 1993; MERCADANTE e LOBO, 1997), que em análises multivariadas os efeitos maternos devem ser incluídos no modelo somente para um caráter, preferentemente G205.

As correlações genéticas encontradas entre G205 e EV, entre 0,71 e 0,86 , demonstram grande associação entre os escores visuais e o crescimento na fase 
Rev. bras. zootec.

Tabela 4 - Covariâncias $^{1}$ (acima da diagonal) e correlações (abaixo da diagonal) para peso ao nascer (PN), ganho de peso do nascimento à desmama ajustado a 205 dias (G205), conformação (CD), precocidade (GD), musculatura (MD) e tamanho (TD) à desmama, a partir de análises multivariadas

Table 4 - Covariances $^{1}$ (above diagonal) and correlations (below diagonal) for birth weight (BW), 205 day adjusted weaning gain (AWG), conformation (WC), precocity (WP), muscling (WM) and size (WS) at weaning, from multivariate analyses

\begin{tabular}{|c|c|c|c|c|c|c|}
\hline Caraterística & PN & G205 & CD & GD & MD & TD \\
\hline Trait & $B W$ & $A W G$ & $W C$ & $W P$ & $W M$ & $W S$ \\
\hline \multicolumn{7}{|c|}{$\begin{array}{l}\text { Efeito genético aditivo direto } \\
\text { Direct additive genetic effect }\end{array}$} \\
\hline $\mathrm{PN}(B W)$ & & $-1,20$ & & & & \\
\hline $\mathrm{G} 205(A W G)$ & $-0,06$ & & 4,54 & 3,87 & 3,99 & 4,16 \\
\hline $\mathrm{CD}(W C)$ & & 0,86 & & 0,16 & 0,14 & 0,14 \\
\hline GD $(W P)$ & & 0,71 & 0,91 & & 0,17 & 0,11 \\
\hline $\mathrm{MD}(W M)$ & & 0,82 & 0,89 & 0,91 & & 0,09 \\
\hline $\mathrm{TD}(W S)$ & & 0,83 & 0,81 & 0,58 & 0,58 & \\
\hline \multicolumn{7}{|c|}{$\begin{array}{l}\text { Efeito genético aditivo materno } \\
\text { Maternal additive genetic effect }\end{array}$} \\
\hline $\mathrm{G} 205(A W G)$ & $-0,05$ & & 0,23 & 0,09 & 0,04 & 0,26 \\
\hline \multicolumn{7}{|c|}{$\begin{array}{l}\text { Efeito de ambiente permanente } \\
\text { Permanent environmental effect }\end{array}$} \\
\hline $\begin{array}{l}\mathrm{PN}(B W) \\
\mathrm{G} 205(A W G)\end{array}$ & 0,99 & 4,42 & 1,83 & 1,84 & 2,26 & 2,26 \\
\hline $\mathrm{CD}(W C)$ & & 1,00 & & 0,07 & 0,08 & 0,08 \\
\hline GD $(W P)$ & & 1,00 & 0,90 & & 0,07 & 0,09 \\
\hline $\mathrm{MD}(W M)$ & & 1,00 & 1,00 & 0,94 & & 0,09 \\
\hline $\mathrm{TD}(W S)$ & & 1,00 & 1,00 & 1,00 & 1,00 & \\
\hline \multicolumn{7}{|c|}{$\begin{array}{l}\text { Efeito de ambiente temporário } \\
\text { Temporary environmental effect }\end{array}$} \\
\hline $\begin{array}{l}\mathrm{PN}(B W) \\
\mathrm{G} 205(A W G)\end{array}$ & 0,02 & 0,98 & 7,66 & 7,15 & 6,79 & 6,73 \\
\hline $\mathrm{CD}(W C)$ & & 0,59 & & 0,42 & 0,45 & 0,35 \\
\hline $\mathrm{GD}(W P)$ & & 0,54 & 0,64 & & 0,41 & 0,25 \\
\hline $\mathrm{MD}(W M)$ & & 0,51 & 0,70 & 0,62 & & 0,27 \\
\hline $\mathrm{TD}(W S)$ & & 0,54 & 0,58 & 0,40 & 0,45 & \\
\hline \multicolumn{7}{|c|}{$\begin{array}{l}\text { Efeito fenotípico } \\
\text { Phenotypic effect }\end{array}$} \\
\hline $\mathrm{PN}(B W)$ & & 3,59 & & & & \\
\hline $\mathrm{G} 205(A W G)$ & 0,04 & & 14,30 & 13,03 & 13,10 & 13,50 \\
\hline $\mathrm{CD}(W C)$ & & 0,69 & & 0,64 & 0,67 & 0,57 \\
\hline $\mathrm{GD}(W P)$ & & 0,61 & 0,72 & & 0,65 & 0,44 \\
\hline $\mathrm{MD}(W M)$ & & 0,63 & 0,76 & 0,71 & & 0,46 \\
\hline $\mathrm{TD}(W S)$ & & 0,66 & 0,66 & 0,50 & 0,53 & \\
\hline
\end{tabular}

${ }^{1} \mathrm{Em} \mathrm{kg}^{2}$ para PN e G205 e em unidades ${ }^{2}$ para CD, GD, MD e TD (In $\mathrm{kg}^{2}$ for $B W$ and $A W G$ and in units ${ }^{2}$ for $W C, W P, W M$ and WS).

pré-desmama. As correlações genéticas entre EV estiveram entre 0,58 e 0,91 . Os valores mais baixos de correlação $(0,58)$ foram encontrados entre GD e TD e MD e TD. A alta correlação entre CD e MD está dentro do esperado, uma vez que o desenvolvimento muscular é considerado na avaliação da conformação do animal e indica que estes escores poderiam ser reunidos num único escore, descrevendo o potencial para a produção de carne do animal como um todo. CARDOSO et al. (1998) também encontraram correlações genéticas altamente positivas entre os mesmos caracteres.

\section{Conclusões}

As magnitudes médias das estimativas de herdabilidade, para os efeitos genéticos direto e total, sugerem que o maior número possível de laços de parentesco deve ser considerado nas análises e os testes de progênie devem ser incentivados para aumentar o progresso genético para G205, CD, GD, MD e TD, na população Angus do RS.

A variação genética aditiva observada para os EV foi semelhante à observada em G205 e de magnitude 
média, indicando que, apesar de terem natureza subjetiva, quando aplicados de maneira criteriosa por avaliadores qualificados, EV são alternativa viável para selecionar e obter ganhos genéticos em caracteres relacionados com a carcaça, como grau de desenvolvimento muscular e grau de acabamento.

É possível aumentar o crescimento na fase prédesmama, sem ocasionar a ocorrência de partos distócicos, pois não existe associação genética importante entre PN e G205.

As associações genéticas positivas entre escores de avaliação visual e entre esses e G205 são favoráveis ao uso de índices de seleção, combinado ganho de peso e escores visuais na fase de desmama.

\section{Referências Bibliográficas}

BEEF IMPROVEMENT FEDERATION - BIF. 1996. Guidelines for uniform improvement programs. Raleigh, NC. 155p.

BOLDMAN, K.G., KRIESE, L.A., VAN VLECK, L.D. et al. 1995. A manual for use of MTDFREML. A set of programs to obtain estimates of variances and covariances [Draft]: U. S. Department of Agriculture, Agricultural Research Service. Lincoln, NE. 120p.

CAMPOS, L.T., CARDOSO, F.F. 1995. Programa de melhoramento de bovinos de carne. Manual do usuário: Associação Nacional de Criadores “Herd Book Collares". Pelotas, RS. 32p.

CARDELLINO, R.A., CARDELLINO M.V. 1984. Herdabilidades dos caracteres de produção à desmama em bovinos Hereford no Rio Grande do Sul. R. Soc. Bras. Zootec., 13(4):557-563.

CARDOSO, F.F. Caracterização genética do desempenho do nascimento à desmama de bovinos Angus criados no Rio Grande do Sul: Pelotas, RS: UFPEL, 1999. 116p. Dissertação (Mestrado) - Universidade Federal de Pelotas, 1999.

CARDOSO, F.F., CARDELliNO, R.A., CAMPOS, L.T. Parâmetros genéticos para escores de avaliação visual à desmama em bovinos da raça Santa Gertrudis. In: REUNIÃO ANUAL DA SOCIEDADE BRASILEIRA DE ZOOTECNIA, 35, 1998, Botucatu. Anais...Botucatu: SBZ, 1998. p.506-508.

ELER, J.P., LOBO, R.B., DUARTE, F.A.M. 1989. Avaliação dos efeitos genéticos direto e materno em pesos de bovinos da raça Nelore criados no Estado de São Paulo. R. Soc. Bras. Zootec., 18(2):112-123.

ELER, J.P., VAN VLECK, L.D., FERRAZ, J.B. et al. 1995. Estimation of variances due to direct and maternal effects for growth traits of Nelore cattle. J. Anim. Sci., 73:3253-3258.

FRIES, L.A. A study of weaning weights in Hereford cattle in the state of Rio Grande do Sul, Brazil: Ames, IA: ISU, 1984. 317p. Thesis (Ph.D.) - Iowa State University, 1984.

FRIES, L.A. Uso de escores visuais em programas de seleção para a produtividade em gado de corte. In: SEMINÁRIO NACIONAL - REVISÃO DE CRITÉRIO DE JULGAMENTO E SELEÇÃO EM GADO DE CORTE. 1996, Uberaba. Anais... Uberaba: ABCZ, 1996. p.1-6.

HOHENBOKEN, W.D., BRINKS, J.S. 1971. Relationships between direct and maternal effects on growth in Herefords: II - Partitioning of covariance between relatives. J. Anim. Sci., 32:26-34.
KOCH, M.R., CUNDIFF, L.V., GREGORY, K.E. 1995. Direct and maternal genetic responses to selection for weaning or yearling weight or yearling weight and muscle score in Hereford cattle. J. Anim. Sci., 73:2951-2958.

KOOTS, K.R., GIBSON, J.P., WILTON, J.W. 1994a. Analyses of published genetic parameters estimates for beef production traits. 1- Heritability. 1994. Anim. Breed Abst., 62(5):309-338.

KOOTS, K.R., GIBSON, J.P., WILTON, J.W. 1994b. Analyses of published genetic parameters estimates for beef production traits. 2- Phenotypic and genetic correlations. Anim. Breed Abst., 62(11):825-853.

MERCADANTE, M.E.Z., LOBO, R.B. 1997. Estimativas de (co)variâncias e parâmetros genéticos dos efeitos direto e materno de características de crescimento de fêmeas de um rebanho Nelore. R. Bras. Zootec., 26(6):1124-1133.

MEYER, K. 1992. Variance components due to direct and maternal effects for growth traits of Australian beef cattle. Livest. Prod. Sci., 31(3-4):179-204.

MEYER, K. 1993. Covariance matrices for growth traits of Australian Polled hereford cattle. Anim. Prod., 57:37-45.

MEYER, K., CARRICK, M.J., DONNELLY, B.J.P. 1993. Genetic parameters for growth traits of Australian beef cattle from a Multibreed selection experiment. J. Anim. Sci.,71:2614-2622.

MOHIUDDIN, G. 1993. Estimates of genetic and phenotypic parameters of some performance traits in beef cattle. Anim. Breed Abst., 61(8):495-522.

PONS, S.B., MILAGRES, J.C., TEIXEIRA, N.M. 1989. Efeitos de fatores genéticos e de ambiente sobre o crescimento e o escore de conformação em bovinos Hereford no Rio Grande do Sul. I - Peso e escore de conformação à desmama. R. Soc. Bras. Zootec., 18(5):391-401.

ROBINSON, D.L. 1996a. Estimation and interpretation of direct and maternal genetic parameters for weights of Australian Angus cattle. Livest. Prod. Sci., 45:1-11.

ROBINSON, D.L. 1996b. Models which might explain negative correlations between direct and maternal genetic effects. Livest. Prod. Sci., 45:111-122.

ROBINSON, D.L., HAMMOND, K., McDONALD, C.A. 1993. Live animal measurements of carcass traits: Estimation of genetic parameters for beef cattle. J. Anim. Sci., 71:1128-1135.

ROSO, V.M., FRIES, L.A. 1995. Componentes principais em bovinos da raça Polled Hereford à desmama e sobreano. $R$. Soc. Bras. Zootec.,24(5):728-735.

SCARPATI, M.T.V., LOBO, R.B., REYES, A. et al. Modelos animais alternativos para estimação de parâmetros genéticos e fenotípicos do peso ao nascer na raça Nelore. In: REUNIÃO ANUAL DA SOCIEDADE BRASILEIRA DE ZOOTECNIA, 35, 1998, Botucatu. Anais...Botucatu: SBZ, 1998. p.514-516.

WILLHAM, R.L. 1972. The role of maternal effect in animal breeding: III. Biometrical aspects of maternal effects in animals. J. Anim. Sci., 35:1288-1302.

WILSON, D.E., BERGER, P.J., WILLHAM, R.L. 1986. Estimates of beef growth trait variances and heritabilities determined from field records. J. Anim. Sci., 63:386-394.

Recebido em: 23/08/99

Aceito em: 24/08/00 